\title{
Caracterização in Situ de Propriedades Mecânicas de Materiais Resistentes ao Desgaste Abrasivo Usando o Método da Indentação
}

\section{(In Situ Determination of Mechanical Properties of Abrasive Wear Resistant Materials Using the Indentation Method)}

\author{
Hans Berns \\ Ruhr-Universität Bochum, Lehrstuhl Werkstofftechnik \\ Universitätstraße 150, 44780, Bochum, Germany
}

\author{
Sinésio D. Franco \\ Universidade Federal de Uberlândia, Laboratório de Tribologia e Materiais \\ Av. Universitária, s/n, Sta. Mônica, 38400-902 - Uberlândia, MG, Brasil
}

\begin{abstract}
Resumo
O desempenho de materiais polifásicos em sistemas tribológicos envolvendo desgaste abrasivo é função de uma série de fatores, a saber: condições de operação, características de projeto e das propriedades do abrasivo e dos microconstituintes do material utilizado. Neste trabalho são apresentados resultados de ensaios de indentação em diferentes microconstituintes e partículas duras, através dos quais as propriedades mais importantes para o fenômeno abrasivo são determinadas in situ. Dentre essas destacam-se a dureza $H$, a tenacidade à fratura $K_{I C}$, o módulo de elasticidade $E$, a relação trabalho plástico / trabalho elástico $W_{p} / W_{e}$ e a relação de durezas entre o microconstituinte e o agente abrasivo. Em muitas situações práticas esses sistemas encontram-se em temperaturas elevadas. Assim, neste trabalho, é também apresentada a influência da temperatura sobre algumas dessas propriedades. Os resultados obtidos mostram a grande potencialidade dessa técnica no processo de seleção e desenvolvimento de materiais resistentes ao desgaste.
\end{abstract}

\section{INTRODUÇÃO}

Os materiais resistentes ao desgaste abrasivo são, normalmente, do tipo polifásicos, contendo uma fase dura $H P$ dispersa numa matriz metálica $M M$. A fase dura é a responsável direta pela resistência ao desgaste. Para ser efetiva no combate a esse tipo de desgaste, as fases duras devem possuir uma dureza superior à dureza do abrasivo $H_{A B}$, e apresentar um tamanho médio maior que a largura dos sulcos produzidos pelo abrasivo dentro da matriz. Os materiais usualmente selecionados para esse fim apresentam durezas, que crescem na seguinte ordem: $H_{M M}<H_{A B}<H_{H P}$. Além disso, a fase dura deve possuir uma boa tenacidade à fratura $K_{I C}$, de modo que o desgaste abrasivo provocado pelo mecanismo de

\begin{abstract}
The wear resistance is not an intrinsic property of materials but depends on the operating conditions, design properties, type of abrasive and material properties. In this work the results of microindentation tests in different hard particles of wear resistant alloys and composites, as well as in bulk materials are presented. Continuous monitoring the load and the indenter penetration depth it is possible to obtain in situ important properties in the wear process of the alloy microconstituents. With this technique it was possible to determine the hardness $H$, the fracture toughness $K_{I C}$, the Young modulus $E$ and the relationship between the plastic and elastic work of deformation $W_{p} / W_{e}$. Since in many practical situations the process temperature is an important parameter, its influence on some of these properties is also considered. The results show that this technique may constitute a helpful tool in the process of selecting suitable microconstituents for wear resistant materials.
\end{abstract}

microlascamento seja evitado [1]. A tenacidade à fratura pode ser determinada através de ensaios de dureza Vickers, desde que cargas suficientemente grandes sejam utilizadas, produzindo-se trincas nas extremidades da impressão de dureza. Conhecendo-se o tamanho dessas trincas, que podem ser do tipo radial ou Palmqvist (figura 1), é possível, então, avaliar essa propriedade. Todavia, a determinação de $K_{I C}$ através desse método está associada ao prévio conhecimento de outras propriedades, como por exemplo, o módulo de elasticidade $E[2,3]$. A caracterização do módulo de elasticidade ou de Young, por sua vez, pode ser efetuada, dentre outras maneiras, através da monitoração e aquisição da carga aplicada e da profundidade de indentação do penetrador em um ensaio de microdureza [4-6]. Além dessas propriedades, $H, K_{I C}$ e $E$, outras 


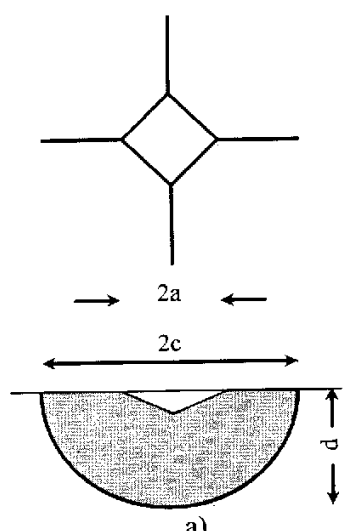

a)

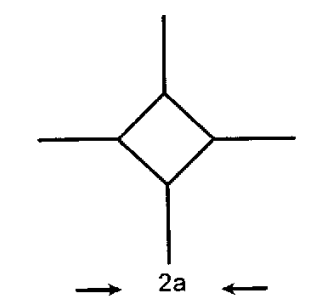

b)

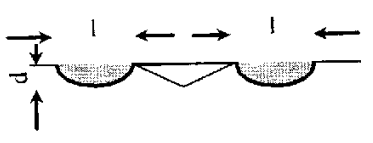

Figura 1: Representação esquemática da geometria de trincas produzidas através da indentação com penetrador Vickers: a) trincas radiais e b) trincas de Palmqvist.

informações dos microconstituintes podem ser obtidas, tais como: a relação entre as energias de deformação plástica e elástica $W_{p} / W_{e}$ relaxação de tensões, trabalho específico de indentação e fluência [7], que também contribuem para a resposta tribológica dos microconstituintes [1]. Como muitos dos microconstituintes utilizados na produção de materiais resistentes ao desgaste abrasivo apresentam tamanho microscópico, a indentação se apresenta como ferramenta importante na caracterização in situ de propriedades mecânicas para a seleção e desenvolvimento de novos materiais.

O presente trabalho tem como objetivo, apresentar a aplicação da técnica de indentação na caracterização in situ de propriedades importantes no desenvolvimento de materiais resistentes ao desgaste abrasivo. Como muitas das aplicações práticas envolvem aumento de temperatura, mostram-se ainda os efeitos deste parâmetro sobre a dureza e os trabalhos para deformação elástica e plástica.

\section{MATERIAIS E MÉTODOS}

Para a realização dos ensaios de microdureza foi utilizado um equipamento com aplicação de carga através de um piezotransladador de alto poder de resolução $(10 \mathrm{~nm})$ [7]. O módulo de elasticidade foi determinado usando a equação A [3,7]

$$
E+\left(1-v^{2}\right) \frac{F_{\text {max }} \cdot \sqrt{2 \pi}}{2 . D\left(h_{t}-h_{c}\right)}
$$

onde: $F_{\text {Max }}$ representa a máxima carga aplicada sobre o penetrador durante o ensaio, $D$ a diagonal da impressão de dureza Vickers e $h_{t}$ e $h_{c}$ a penetração total sob a ação da força máxima e após a retirada da carga, respectivamente (figura 2). A tenacidade à fratura, por sua vez, foi determinada usando a equação sugerida por Liang et al. [3], descrita por B

$$
\left.\left(\frac{\mathrm{K}_{\mathrm{IC}} \cdot \emptyset}{\mathrm{H} \cdot \mathrm{a}^{1 / 2}}\right)\left(\frac{\mathrm{H}}{\mathrm{E} . \varnothing}\right)^{0,4} \quad \alpha=\left(\frac{\mathrm{c}}{\mathrm{a}}\right)^{\left(\frac{\mathrm{c}}{18 \cdot \mathrm{a}}-1,51\right.}\right)
$$

onde: $H$ representa a dureza Vickers, $E$ o módulo de elasticidade, $\mathrm{f}$ um fator de constrição ( $\mathrm{f}=\mathrm{H} / \mathrm{s}_{\mathrm{y}}$ » $3, \mathrm{~s}_{\mathrm{y}}$ - tensão de escoamento), $a$ a metade da impressão de dureza e $c$ a metade da trinca produzida pelo processo de indentação (figura 2). O parâmetro $\alpha$ é dado através da equação $\mathrm{C}$

$$
\alpha=14\left[1-8\left(\frac{4 . v-0,5}{1+v}\right)^{4}\right]
$$

onde $\mathrm{n}$ representa o coeficiente de Poisson.

Dentre as várias fases duras presentes em ligas ou compostos de matriz metálica ou de matriz cerâmica resistentes ao desgaste abrasivo foram testados carbetos, boretos e óxidos, tais como: $\mathrm{WC} /$ $\mathrm{W}_{2} \mathrm{C}$ de forma esférica e fragmentada, WC sinterizado, $\mathrm{NbC}, \mathrm{CrB}_{2}$, $\mathrm{Ni}_{3} \mathrm{~B}, \mathrm{M}_{7} \mathrm{C}_{3}, \mathrm{M}_{3} \mathrm{C}, \mathrm{Al}_{2} \mathrm{O}_{3}$ e $\mathrm{SiC}$. Essas partículas foram embutidas em matrizes metálicas por prensagem isostaticamente a quente (HIP), resultando em Compostos de Matriz Metálica (MMC) ou, utilizando cerâmicas endurecíveis a baixas temperaturas, formando assim, Compostos de Matriz Cerâmica (CMC). Os NbC, $\mathrm{M}_{3} \mathrm{C}$ e $\mathrm{M}_{7} \mathrm{C}_{3}$ foram testados em amostras de superligas de níquel e ferros fundidos brancos. Apresentam-se ainda resultados de indentação obtidos em nitreto cúbico de boro policristalino (PCBN) utilizados na confecção de ferramentas de usinagem de alto rendimento (tabela I).

As superfícies das amostras testadas foram preparadas através de metalografia convencional usando pasta de diamante de até $1 \mu \mathrm{m}$ e, por fim, pasta de $\mathrm{Al}_{2} \mathrm{O}_{3}$ de $0,2 \mu \mathrm{m}$. Os valores de $\mathrm{K}_{\mathrm{IC}}$ foram obtidos através da medição das trincas em pelo menos 10 indentações em microscópio ótico.

\section{RESULTADOS E DISCUSSÃO}

Os resultados dos ensaios de microdureza em função da temperatura são apresentados na figura 3 para algumas das partículas duras mais comuns em materiais resistentes ao desgaste abrasivo. Observa-se dessa figura, à temperatura ambiente, uma considerável dispersão dos resultados para as diferentes partículas duras. Comparando-se esses resultados com os da literatura, obtidos através de microdurômetro convencional, tem-se uma boa correlação de valores [8]. As pequenas diferenças existentes podem ser atribuídas a variações de composição, uma vez que os ensaios efetuados nesse trabalho foram conduzidos segundo normas de dureza da ASTM. Das partículas duras estudadas, o carbeto fundido de tungstênio (fragmentado) apresenta o maior valor de dureza

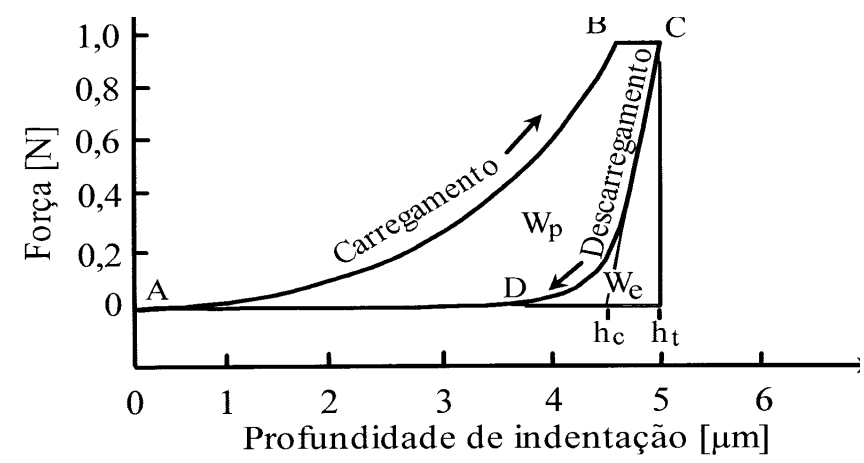

Figura 2: Curvas da força em função da profundidade de indentação para a fase dura $\mathrm{Ni}_{3} \mathrm{~B}$ a $800^{\circ} \mathrm{C}, \mathrm{BC}$ - fluência sobre $10 \mathrm{~s}$. 
Tabela I: Nitretos cúbicos de boro policristalinos estudados.

\begin{tabular}{llll}
\hline PCBN & $\begin{array}{l}\text { Diâmetro médio } \\
(\mu \mathrm{m})\end{array}$ & $\begin{array}{l}\text { Composição da matriz } \\
(\% \text { em peso) }\end{array}$ & $\begin{array}{l}\text { HV0,05 } \\
\text { (Temperatura ambiente) }\end{array}$ \\
\hline PCBN1 & $5-15$ & $71 \mathrm{Al}, 29 \mathrm{Si}$ & 4779 \\
\hline PCBN2 & $2-5$ & $50 \mathrm{Co}, 13 \mathrm{~W}, 29 \mathrm{Al}, 8(\mathrm{Fe}, \mathrm{Ni})$ & 5118 \\
\hline PCBN3 & $1-4$ & $66 \mathrm{Ti}, 5 \mathrm{~W}, 27 \mathrm{Al}, 2 \mathrm{Fe}$ & 3706 \\
\hline PCBN4 & $0,5-1,5$ & $81 \mathrm{Ti}, 6 \mathrm{~W}, 13 \mathrm{Al}$ & 3797 \\
\hline
\end{tabular}

(2562 $\pm 312 \mathrm{HV} 0,05)$, que pode ser atribuído ao fino eutético de WC e $\mathrm{W}_{2} \mathrm{C}$. Devido ao maior grau de refino do carbeto fundido de tungstênio esférico, um maior valor de dureza foi verificado nessa partícula dura $(2989 \pm 167 \mathrm{HV} 0,05)$. O menor valor de dureza foi encontrado na cementita, que compõe os ferros fundidos brancos empregados com grande freqüência em sistemas sujeitos a desgaste abrasivo. $\mathrm{O}$ emprego dessa $H P$ em componentes sujeitos a esse tipo de desgaste se justifica pelo baixo preço dos ferros fundidos brancos não ligados ou de baixo teor em cromo e pelo fato de que, nesses tribosistemas o óxido de silício (flint) se apresenta como um dos principais elementos abrasivos. Como o flint apresenta uma dureza média de $1100 \mathrm{HV} 0,05$ já à temperatura ambiente, tem-se, então, uma dureza da partícula dura superior à do abrasivo, ou seja, $H_{H P} / H_{\text {Flint }}>1$ (figura 4). Dessa forma, a cementita pode atuar de forma efetiva no aumento da resistência ao desgaste abrasivo.

Com o aumento da temperatura, a dureza de todas as partículas duras decresce (observar escala logarítmica no eixo da dureza), podendo ou não apresentar um ponto de inflexão que, usualmente, corresponde à metade da temperatura de fusão $[9,10]$. Acima dessa temperatura, a dureza decresce rapidamente, como pode ser verificado nos carbetos de cromo do tipo $\mathrm{Cr}_{2} \mathrm{C}_{3}$. O não aparecimento desse ponto em partículas como $\mathrm{NbC}$ se deve ao fato de que a metade da temperatura de fusão, neste caso, se encontra acima da faixa de temperaturas estudadas. O WC representa uma exceção, onde a partir de $600{ }^{\circ} \mathrm{C}$ a dureza permanece praticamente constante. Esse comportamento parece, segundo Chatfield [11], estar associado ao tamanho de grão do WC.

Relacionando-se esses valores de dureza das partículas du-

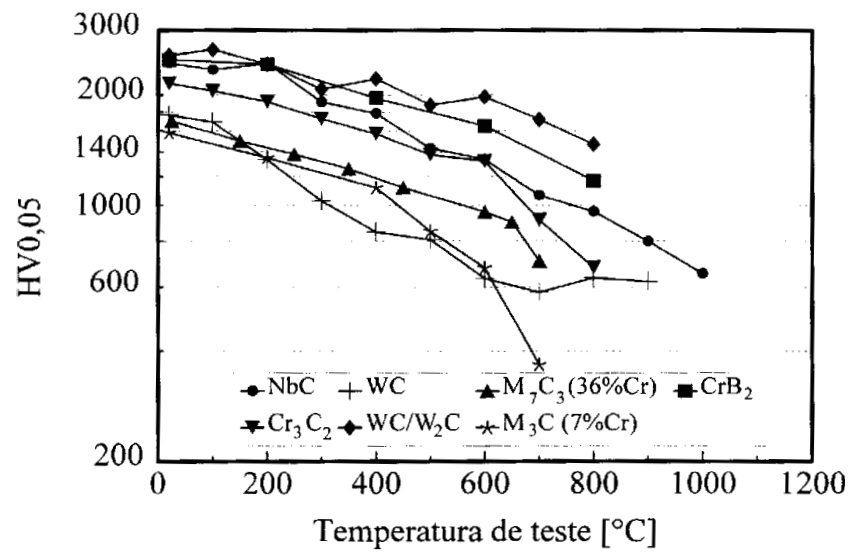

Figura 3: Microdureza a quente de algumas das partículas duras mais utilizadas em materiais resistentes ao desgaste abrasivo, $M_{7} C_{3}$ segundo Kagawa et al.[9]. ras com a dureza do flint $H_{H P} / H_{\text {Flint }}$ em função da temperatura, notase que essa relação cresce continuamente. Portanto, em aplicações a quente, a utilização dessas partículas duras num tribosistema contendo como abrasivo flint, é de se esperar, em princípio, uma redução na agressividade do abrasivo, uma vez que a partícula dura passa a ser bem mais dura do que o abrasivo. Além disso, há um incremento de tenacidade à fratura, que tenderia a reduzir a probabilidade de ocorrência de microlascamento e, portanto, melhorando a resistência ao desgaste com o aumento da temperatura.

Como a dureza representa um importante parâmetro no comportamento tribológico de ferramentas de usinagem são apresenta-

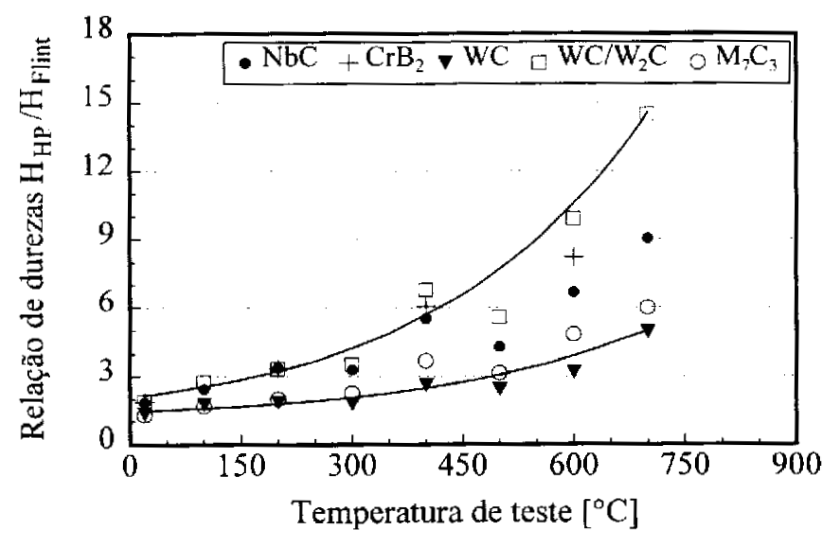

Figura 4: Relação de durezas entre partículas duras e flint $H_{H P} / H_{\text {Flint }}$ em função da temperatura de teste.

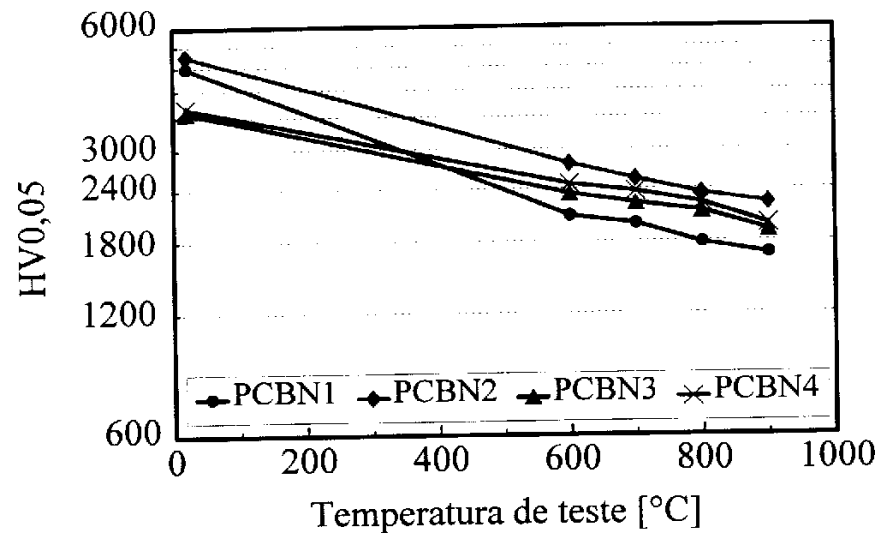

Figura 5: Dureza em função da temperatura de teste para nitretos cúbicos de boro policristalinos. 
Tabela II: Trabalho de deformação e módulo de Young obtidos através da técnica de microindentação.

\begin{tabular}{|c|c|c|c|c|c|c|}
\hline Material & $\begin{array}{l}\text { Temp. } \\
\left({ }^{\circ} \mathrm{C}\right)\end{array}$ & $\begin{array}{l}\mathrm{E} /\left(1-v^{2}\right) \\
(\mathrm{GPa})\end{array}$ & $\begin{array}{l}\mathrm{W}_{\mathrm{pl}} \\
(\mu \mathrm{J})\end{array}$ & $\begin{array}{l}\mathrm{W}_{\mathrm{el}} \\
(\mu \mathrm{J})\end{array}$ & $\begin{array}{l}\mathrm{W}_{\mathrm{H}} \\
\left(\mathrm{J} \mathrm{mm}^{-3}\right)\end{array}$ & $\mathrm{W}_{\mathrm{pl}} / \mathrm{W}_{\mathrm{el}}$ \\
\hline \multirow[t]{3}{*}{$\mathrm{NiCr} 10$} & 20 & 237 & 1,83 & 0,17 & 2,0 & 10,8 \\
\hline & 394 & - & 2,10 & 0,16 & 1,7 & 13,1 \\
\hline & 753 & - & 2,55 & 0,17 & 1,1 & 15 \\
\hline \multirow[t]{3}{*}{$\mathrm{Ni}_{3} \mathrm{~B}$} & 20 & 135 & 0,35 & 0,51 & 11,8 & 0,69 \\
\hline & 421 & 123 & 0,60 & 0,58 & 7,5 & 1,03 \\
\hline & 791 & 102 & 1,99 & 0,44 & 2,2 & 4,52 \\
\hline \multirow[t]{3}{*}{$\mathrm{SiC}$} & 20 & 385 & 0,11 & 0,31 & 41,8 & 0,35 \\
\hline & 390 & 289 & 0,27 & 0,37 & 26,5 & 0,73 \\
\hline & 815 & 207 & 0,36 & 0,40 & 17,1 & 0,90 \\
\hline \multirow[t]{4}{*}{ PCBN4 } & 20 & 428 & 0,05 & 0,17 & & 0,33 \\
\hline & 400 & 307 & 0,11 & 0,18 & & 0,59 \\
\hline & 800 & 179 & 0,24 & 0,21 & & 1,15 \\
\hline & 1.000 & 165 & 0,23 & 0,21 & & 1,09 \\
\hline \multirow[t]{5}{*}{ PCBN2 } & 20 & 414 & 0,06 & 0,19 & & 0,34 \\
\hline & 400 & 291 & 0,44 & 0,41 & & 1,06 \\
\hline & 600 & 245 & 0,67 & 0,55 & & 1,22 \\
\hline & 800 & 189 & 1,12 & 0,39 & & 2,83 \\
\hline & 1.000 & 166 & 1,13 & 0,34 & & 3,31 \\
\hline $\mathrm{CFT}^{*}$ & 20 & 659 & & & & 1,74 \\
\hline $\begin{array}{l}\mathrm{M}_{3} \mathrm{C} \\
\mathrm{M}=14 \% \mathrm{Cr}^{* *} 2\end{array}$ & 20 & 329 & & & & 1,92 \\
\hline $\begin{array}{l}\mathrm{M}_{7} \mathrm{C}_{3} \\
\mathrm{M}=41 \% \mathrm{Cr}^{* *}\end{array}$ & 20 & 367 & & & & 2,87 \\
\hline
\end{tabular}

* - carbeto fundido de tungstênio, ** restante: ferro.

dos também, através da figura 5, o efeito da temperatura sobre essa propriedade em nitretos cúbicos de boro policristalinos com diferentes matrizes. Nota-se através dessa figura (escala logarítmica na dureza) um decréscimo linear da dureza em toda a faixa de temperatura estudada. Merece destaque ainda nessa figura os elevados valores de dureza apresentados pelo PCBN2 em altas temperaturas (superior a $2000 \mathrm{HV} 0,05$ a $900{ }^{\circ} \mathrm{C}$ ), o que torna essa classe de material atraente para fins de usinagem e trabalho a quente.

Os resultados da avaliação da recuperação elástica, substituídos na equação A, permitiram a obtenção de módulos de elasticidade $E$ de partículas duras, cujos desvios padrões foram inferiores a $10 \%$, correspondendo a valores usuais obtidos em outros equipa-

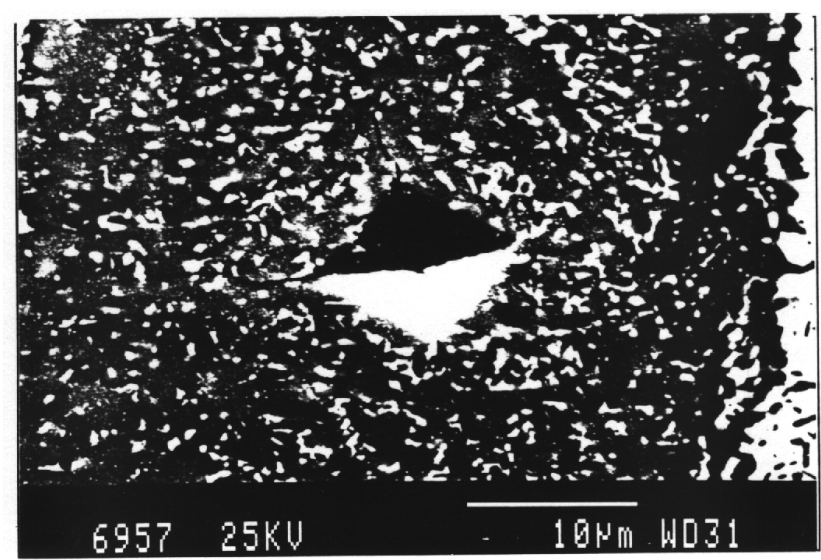

Figura 6: Micrografia mostrando a formação de trincas nos cantos da impressão de dureza HV0,05, MEV.

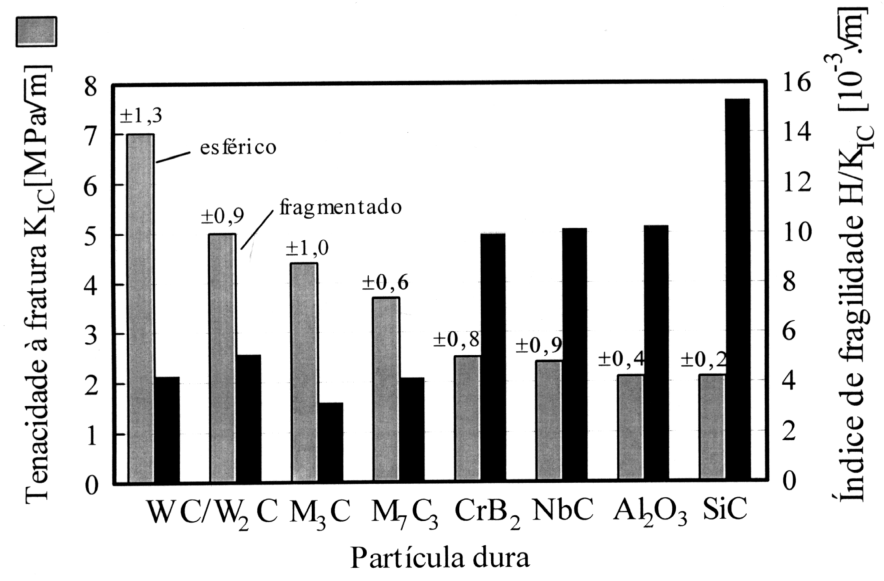

Figura 7: Tenacidade à fratura e índice de fragilidade de algumas partículas duras à temperatura ambiente. $\mathrm{No} \mathrm{M}_{7} \mathrm{C}_{3} \mathrm{M}=41 \% \mathrm{Cr}$ e no $\mathrm{M}_{3} \mathrm{C} \mathrm{M}=14 \% \mathrm{Cr}$.

mentos do mesmo gênero [4]. Outros resultados da determinação de propriedades mecânicas, bem como o efeito da temperatura sobre o módulo de elasticidade podem ser obtidos na referência [2].

Conhecido $E$, determinou-se a tenacidade à fratura, após a medição do comprimento das trincas geradas nos cantos das impressões de dureza (figura 6). A figura 7 apresenta os valores de $K_{I C}$ para partículas duras estudadas à temperatura ambiente. Desses valores pode-se observar, que o carbeto fundido de tungstênio apresenta uma excelente combinação de tenacidade à fratura e dureza, podendo conduzir a enormes reduções da taxa de desgaste abrasivo, principalmente na forma esférica, que corresponde ao eutético mais refinado. Essas expectativas foram posteriormente confirmadas através de ensaios de desgaste abrasivo de compostos de matriz metálica $M M C s$ com diferentes tipos de partículas duras [2, 12]. Valores de tenacidade à fratura relativamente baixos puderam ser observados em abrasivos como $\mathrm{Al}_{2} \mathrm{O}_{3}$ e SiC.

Além das propriedades descritas acima, o controle do processo de indentação e a aquisição da curva de força em função da profundidade permite o cálculo de outros parâmetros, a saber: trabalhos elástico e plástico de deformação e o trabalho específico no processo em função da temperatura, como mostrado na tabela II. Colocando-se os resultados $W_{p} / W_{e}$ em uma escala $\log \mathrm{x}$ $\log$ (figura 8), nota-se que os diversos materiais testados através 


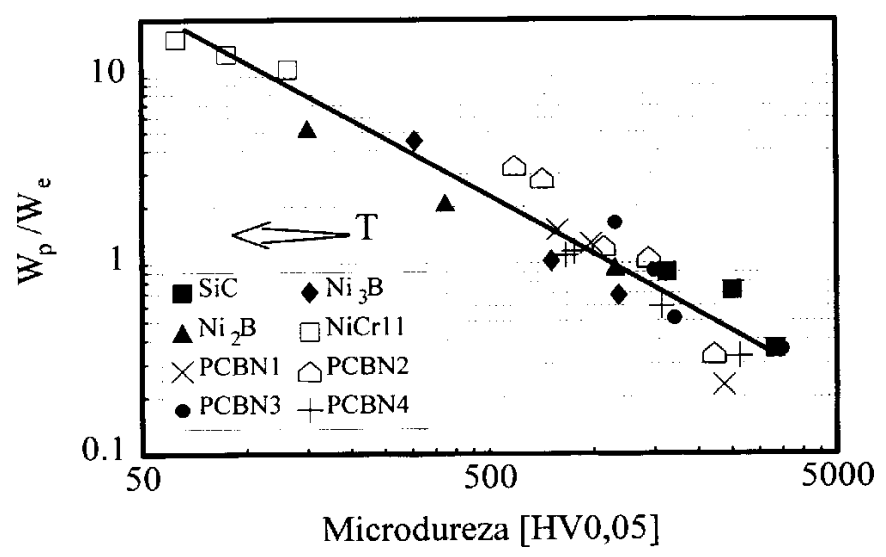

Figura 8: Evolução da relação entre o trabalho de deformação plástica e elástica de indentação em função da temperatura e da dureza para partículas duras e PCBNs.

dessa técnica, fornecem uma relação de $W_{p} / W_{e}$ em função da temperatura ou microdureza do tipo linear. Isso se deve, basicamente, ao aumento de ductilidade dos materiais com a temperatura. Aumenta-se assim, a deformação plástica durante o processo de indentação.

\section{CONCLUSÕES}

A precisão e resolução do equipamento utilizado para testes em temperaturas de até $1000{ }^{\circ} \mathrm{C}$ permitem a determinação in situ da dureza, do módulo de elasticidade, da tenacidade à fratura, dos trabalhos elástico e plástico no processo de indentação, bem como o trabalho específico dos diversos microconstituintes em materiais polifásicos. A técnica mostrou ser uma ferramenta muito importante no processo de seleção e desenvolvimento de materiais resistentes ao desgaste, inclusive quando a temperatura tiver de ser considerada.

\section{REFERÊNCIAS}

[1] K.H. Zum Gahr; "Microstructure and wear of materials", Amsterdam, Elsevier, 1987.

[2] H. Berns e S.D. Franco; "Properties of hard particles at room and elevated temperature", III Seminário Brasileiro de Materiais Resistentes ao Desgaste, Fortaleza, 1994, p.131-146.

[3] K.M. Liang, G. Orange e G. Fantozzi; "Evaluation by indentation of fracture toughness of ceramic materials", J. Mater. Sci., 25(1990)207-214.

[4] B.N. Lucas, W.C. Oliver, R.K. Williams, J. Brynestad e M.E. O'Hern; "The hardness and Young's modulus of bulk $\mathrm{YBa}_{2} \mathrm{Cu}_{3} \mathrm{O}_{7}$ $(1: 2: 3)$ and $\mathrm{YBa}_{2} \mathrm{Cu}_{4} \mathrm{O}_{8}(1: 2: 4)$ as determined by ultra low load indentation", J. Mater. Res., 6(1991)2519-2522.

[5] M.L. Emiliani; "Debond coating requirements for brittle matrix composites”, J. Mater. Sci., 28(1993)5280-5296.

[6] R. Nowak e M. Sakai; "Energy principle of indentation contact: The application to sapphire", J. Mater. Res., 8(1993)1068-1078.

[7] M. Lührig; "Temperaturabhängigkeit der Mikrohärte von Mischkristallen in Phasengemischen", Dr. Ing. Thesis, RuhrUniversität Bochum, 1992.

[8] G.V. Samsonov; "High-temperature materials", Band 2, Plenum Press, New York, 1964.

[9] A. Kagawa, T. Okamoto, K. Saito e M. Ohta; "Hot hardness of ( $\mathrm{Fe}, \mathrm{Cr})_{3} \mathrm{C}$ and $(\mathrm{Fe}, \mathrm{Cr})_{7} \mathrm{C}_{3}$ carbides", J. Mater. Sci., 19 (1984) 2546-2554. [10] Y. Kumashiro, Y. Nagai e H. Kato; "The Vickers micro-hardness of $\mathrm{NbC}, \mathrm{ZrC}$ and $\mathrm{TaC}$ single crystals up to $1500^{\circ} \mathrm{C}$ ", J. Mater. Sci. Lett., 1(1982)49-52.

[11] Ch. Chatfield; "The influence of carbide grain size on the hot hardness of polycrystalline tungsten carbide and WC-Co cemented carbides", Powder Met. Int., 17(1985)113-115.

[12] S.D. Franco; "Wechselwirkung zwischen Matrix und Hartphasen beim Warmverschleiß”, Dr.Ing. Thesis Ruhr-Univ. Bochum, 1995.

(Rec. 9/97, Ac. 12/97) 